\title{
Stratégies d'élaboration, de partage et de réutilisation de scénarios pédagogiques
}

\section{Valérie Emin}

Institut national de recherche pédagogique - Équipe EducTice - Lyon

et Laboratoire Informatique de Grenoble - FRANCE

valerie.emin@inrp.fr

\section{Jean-Philippe Pernin}

Institut national de recherche pédagogique - Équipe EducTice - Lyon

et Laboratoire Informatique de Grenoble - FRANCE

jean-philippe.pernin@inrp.fr

\section{Michèle Prieur}

Institut national de recherche pédagogique - Équipe ACCES - Lyon - FRANCE

michele.prieur@inrp.fr

\section{Eric Sanchez}

Institut national de recherche pédagogique - Équipe EducTice et LEPS - Université de Lyon - FRANCE

eric.sanchez@inrp.fr

\section{Scénariser, un acte pédagogique}

\section{Résumé}

Cet article s'intéresse à la création, au partage et à la réutilisation de scénarios pédagogiques. Notre contribution se base sur l'analyse des activités de scénarisation menées par deux enseignants experts s'inscrivant dans une démarche de recherche en didactique des sciences. Ces enseignants ont élaboré et testé plusieurs versions de scénarios mettant en œuvre une démarche d'investigation et précisant l'organisation des activités à réaliser par les élèves et les enseignants. À partir de l'analyse effectuée, nous tentons de dégager un modèle intégrant les dimensions didactique, pédagogique ou situationnelle dans l'élaboration des scénarios, et proposons quelques pistes pour le partage et la réutilisation de ces scénarios.

\section{Abstract}

This paper focuses on designing and reusing of learning scenarios. Our contribution is based on the analysis of scenarisation activities led by two expert teachers involved in an educational research program in didactics of sciences. These practitioners have elaborated various versions of scenarios implementing an investigating approach and specifying the organisation of activities to be done by students and teachers. From the performed analysis, we try to isolate a model integrating the didactical, pedagogical and situational dimensions in the design of learning scenarios; we also propose some reflexions about sharing and reusing of these scenarios.

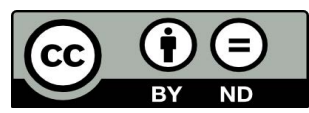

(C)Auteur(s). Cette œuvre, disponible à http://www.ritpu.org/IMG/pdf/ritpu0402_emin.pdf, est mise à disposition selon les termes de la licence Creative Commons Attribution - Pas de Modification 2.5 Canada : http://creativecommons.org/licences/by-nd/2.5/ca/deed.fr 


\section{Introduction}

Ce travail s'inscrit dans le cadre du projet CAUSA (Conceptions, analyses et usages des scénarios d'apprentissage), mené depuis 2005 au sein de l'INRP (Institut national de recherche pédagogique). Ce projet se donne pour objectif de proposer aux praticiens des modèles et des outils leur permettant de concevoir, mettre en place, suivre, adapter, analyser et mutualiser les scénarios dans le cadre de l'enseignement académique. Dans une première phase exploratoire, nous avons effectué un travail de recensement des représentations et des pratiques des enseignants (Pernin et Emin, 2006). Parmi les résultats dégagés, nous avons pu constater l'importance de la réutilisation de scénarios existants au sein de communautés d'enseignants. À partir de ce constat, nous désirons analyser plus finement les stratégies et les intentions sous-jacentes aux scénarios. Cette contribution s'appuie sur une expérimentation concernant l'organisation de séquences pédagogiques en Sciences de la Vie et de la Terre dans l'enseignement secondaire français. Cette expérimentation, effectuée dans le cadre de travaux de recherche en didactique des sciences, vise à proposer et à tester des modalités de mise en œuvre d'une démarche d'investigation en sciences basée sur l'utilisation conjointe de différents outils numériques et comprenant un ensemble de modalités variées (travail en classe, à la maison, sur le terrain). L'objectif de cette contribution est de démontrer que les scénarios mis en œuvre correspondent à une démarche type appliquée à des contextes spécifiques; il s'agit ici d'une démarche d'investigation scientifique en Sciences de la Vie et de la Terre proche de celle décrite par les programmes officiels publiés par le ministère de l'Éducation nationale. Les contextes tiennent notamment compte des particularités liées à la discipline (ici la géologie), ainsi que des contraintes organisationnelles, spatiales, temporelles, techniques et économiques dans lesquelles s'inscrivent les activités. À partir de cette analyse, nous tentons de dégager un modèle précisant les places respectives des dimensions didactique, pédagogique ou situationnelle dans l'élaboration des scénarios pédagogiques.

Cet article est organisé de la façon suivante : dans la section 1, nous présentons le contexte dans lequel s'est déroulée l'expérimentation et nous décrivons de façon plus précise deux scéna- rios abordant des thématiques disciplinaires proches. Dans la section 2, nous étudions le processus d'élaboration de ces scénarios en insistant en particulier sur les différences de contexte qui ont conduit les mêmes concepteurs à proposer des solutions variées. Dans la section 3, nous proposons un processus générique de conception en deux phases reposant sur l'expression des intentions puis sur l'affinage progressif du scénario. Dans la section 4, nous soulevons des questions relatives au partage de ces scénarios à partir desquelles pourraient être mises en œuvre de nouvelles stratégies de réutilisation. Enfin, en conclusion, nous traçons quelques perspectives sur la poursuite de nos travaux.

\section{Description de l'expérimentation Géonote}

Géonote (Lefèvre et Sanchez, 2006) est un environnement informatique conçu et développé au sein de l'INRP. Ce logiciel, destiné à l'enseignement des sciences de la Terre dans l'enseignement secondaire, permet de consulter des images géoréférencées sur une carte et d'effectuer un ensemble d'opérations de traitement de l'information spatiale telles que la fusion de couches d'information topographique et géologique, la mesure de distances sur la carte ou la localisation géographique d'objets géologiques. Un mode d'édition permet également de géoréférencer des images commentées sur un secteur géographique donné. Après les phases de conception participative et de mise au point du logiciel, deux séries d'expérimentations, s'inscrivant dans une démarche d'ingénierie didactique (Artigue, 1988), ont été menées en 2005 et 2006 dans quatre classes de collège et cinq classes de lycée. Il s'agissait, d'une part, d'éprouver les hypothèses de recherche de nature didactique concernant la mise en œuvre d'une démarche d'investigation en sciences de la Terre et, d'autre part, d'évaluer l'utilisabilité, l'acceptabilité et l'utilité du logiciel (Tricot et al., 2003). Afin de cadrer le travail à réaliser par les différents acteurs (élèves et enseignants), des scénarios décrivant précisément les tâches à réaliser ont été définis. Nous présentons ici deux de ces scénarios en indiquant les tâches prescrites, les ressources utilisées ainsi que le contexte et la durée dans lesquels s'inscrivent ces tâches.

Le premier scénario concerne une expérimentation réalisée auprès d'élèves de terminale scientifique devant répondre 
Phase 1 : préparation de la classe de terrain (séance de TP de $2 \mathrm{~h}$ et travail à la maison) L'objectif de cette phase est l'appropriation par les élèves du modèle de collision des plaques lithosphériques et l'élaboration d'un protocole d'observation de façon à ce que les élèves se rendent sur le terrain en sachant les observations à réaliser, les informations à recueillir et les mesures à effectuer.

Ce que doivent faire les elèves...

- identifier les phénomènes géologiques impliqués dans la formation d'une chaîne de collision et les traduire en traces géologiques observables sur le terrain ;

- localiser les sites géologiques à étudier, les traces témoins d'une collision à rechercher et déterminer les itineraires à parcourir ;

- déterminer les mesures à effectuer et les obserwations à effectuer.

Ce dont disposent les elèves...

Un schéma représentant un modèle scientifique décrivant la formation d'une chaîne de collision

Geonote (secteurs géographiques du

Che naillet et du Briançonnais)

Site de calcul d'itinéraire routier

Formulaire de consignes accessibles en ligne

Phase 2 : classe de terrain (excursion de 2 jours)

L'objectif de cette classe de terrain est la collecte des données géologiques et un premier traitement de ces données.

Ce que doivent faire les élèves..

- échantillonner, rechercher, identifier, localiser. photographier, orienter, cartographier, mesurer, modéliser les traces montrant que les Alpes sont une chaîne de collision.

Ce dont disposent les élèves...
GPS, appareil photo numérique, carte
topographique, panorama, loupe,
photographie aérienne, boussole, pâte
à modeler...

Phase 3 : exploitation de la classe de terrain (séance de TP de 2 h) L'objectif de cette séance est le traitement des données collectées sur le terrain de façon à proposer une réponse argumentée au problème posé.

Ce que dovent faire les éleves..

- sélectionner, mettre en forme leurs photographies; dans

Géonote, géoréférencer et commenter ces images sur un

secteur géographique;

- rédiger une histoire argumentée des Alpes dans le bloc-

notes de Géonote.

Ce dont disposent les élèves...

Géonote, photographies et autres

informations recueillies sur le terrain,

bloc-notes

Formulaire de consignes accessibles en ligne

Figure 1. Scénario de la classe de terrain du Chenaillet

au problème suivant : "En quoi les Alpes sont-elles une chaîne de collision? ». Ce scénario se fonde principalement sur une confrontation directe des élèves aux données observées et collectées sur le terrain, en l'occurrence le massif du Chenaillet (Hautes-Alpes - France); il comprend trois phases articulant activités de classe, travail de terrain et activités réalisées à la maison.

Un second scénario mettant en œuvre une démarche d'investigation similaire a été expérimenté auprès d'élèves de quatrième de collège. Ayant au préalable étudié différents modèles de frontières lithosphériques (accrétion, collision, subduction), les élèves ont pour objectif de comprendre le fonctionnement des plaques lithosphériques dans la région du lac Baikal (Russie). Ils doivent choisir parmi les trois modèles étudiés celui correspondant à la région considérée.

Au regard du précédent, ce scénario présente des spécificités : l'éloignement du site étudié exclut un déplacement sur le terrain, l'accès direct et privilégié au réel a été remplacé par un ensemble d'activités réalisées en classe. Ainsi, grâce au logiciel Géonote, l'élève peut consulter un jeu d'images géoréférencées de la région étudiée. La phase d'exploration s'en trouve modifiée, tant par la nature « virtuelle » des outils mis en œuvre que par sa durée fortement réduite. Par ailleurs, ce second scénario a nécessité un cadrage particulier lié à la nature du public. En premier lieu, les modèles de la tectonique des plaques étant étudiés pour la première fois en classe de quatrième, une séance entière a été consacrée 


Phase 1 : élaborer une synthèse sur les modèles de fonctionnement
des plaques lithosphériques (cours 1 heure)

Figure 2. Scénario de l'étude de données de terrain sur le lac Baikal

à l'appropriation de ces modèles. En second lieu, le logiciel Géonote a été paramétré de façon particulière pour limiter le nombre d'informations à traiter par un élève de collège pour chaque site, l'élève n'ayant accès qu'à une seule donnée géoréférencée et à un seul document annexe. Cependant, les grandes étapes du scénario et les objectifs des tâches proposées présentent de fortes similarités avec ceux du scénario conçu pour des élèves de terminale pour une classe de terrain. Nous nous proposons d'analyser plus précisément le processus d'élaboration de ces deux scénarios.

\section{Le processus d'élaboration des scénarios expérimentés}

Ce processus s'est appuyé sur une première phase pendant laquelle ont été effectués des choix déterminants en matière de démarche d'apprentissage, associés à un travail de repérage des compétences nécessaires à l'acquisition des connaissances visées. Dans un second temps, le scénario définissant le découpage en séances et l'enchaînement des tâches prescrites a été réalisé sous la conduite d'un ensemble de contraintes relatives au contexte de mise en œuvre.

\subsection{Le cadre général : une démarche d'investigation scientifique}

Dans une publication précédente (Sanchez et Prieur, 2006), il a été montré que l'élaboration des scénarios que nous décrivons ici était une conséquence directe de la mise en œuvre d'une démarche d'investigation scientifique. Cette démarche repose sur la confrontation d'un modèle explicite et d'un registre empirique constitué par l'élève lors d'activités de laboratoire ou de terrain. Elle s'appuie sur les travaux qui définissent les modèles scientifiques comme des intermédiaires entre les aspects théo- 


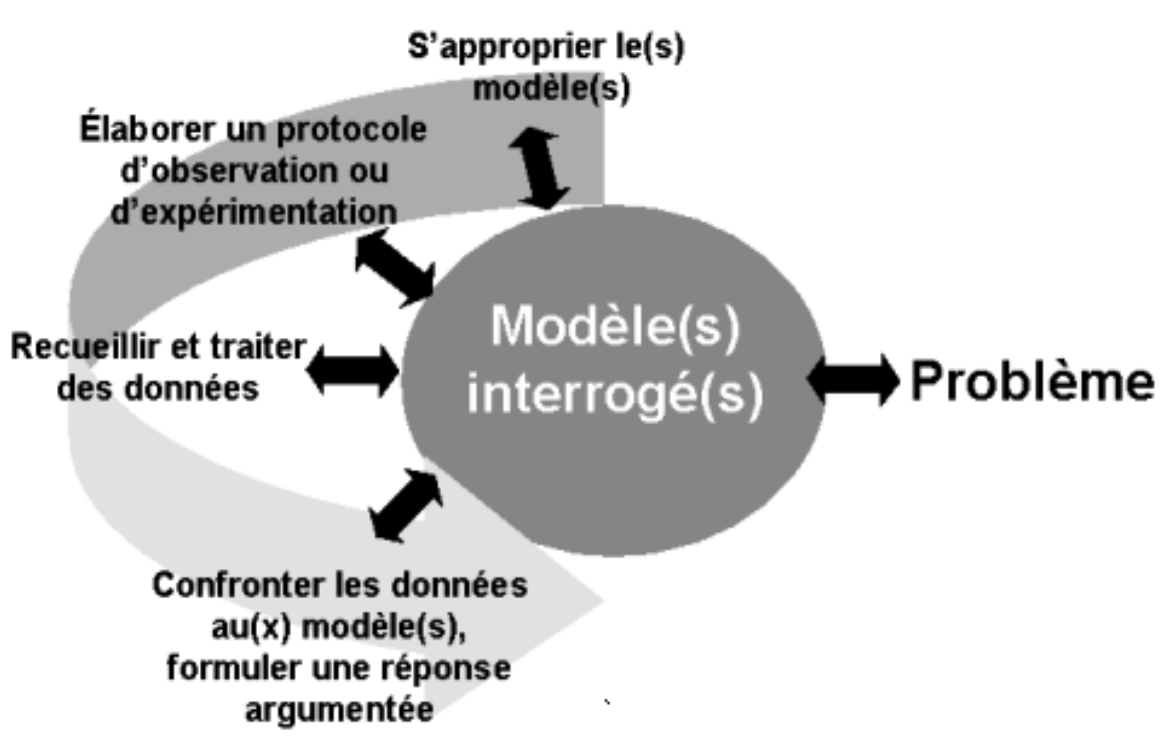

Figure 3. Les quatre phases d'une démarche d'investigation scientifique

Ces différentes phases (appropriation du modèle, élaboration d'un protocole, expérimentation/ observation, formulation d'une réponse) s'articulent autour du (des) modèle(s) scientifique(s) mis en jeu et clairement explicité(s) et ne s'enchaînent pas nécessairement de façon séquentielle. Des allers-retours sont possibles. Elles imposent cependant des contraintes de rythme et sont associées à la mise en œuvre d'une démarche type pouvant donner lieu à un ensemble de déclinaisons différentes. Les scénarios créés diffèreront ainsi par des critères tels que le

riques et perceptifs d'une réalité (Bachelard, 1979; Bunge, 1975; Halloun, 2004; Walliser, 1977). Ce point de vue exclut les activités pour lesquelles l'enseignant fournit à l'élève toutes les informations utiles pour répondre à une question donnée. Une telle démarche d'investigation correspond donc à l'emploi d'un ou plusieurs modèles scientifiques pour interpréter un registre empirique et à leur utilisation pour résoudre un problème ouvert. Elle conduit l'élève à établir des relations entre une réalité soumise à investigation et un modèle scientifique. Ainsi, la mise en œuvre d'une démarche d'investigation scientifique peut être décrite par l'articulation de quatre grandes phases s'articulant autour d'une situation problème « interrogeant » un modèle :

- Une phase initiale consiste pour les élèves en un travail d'appropriation du modèle afin que celui-ci prenne le statut $\mathrm{d}^{\prime} \ll$ outil pour penser »;

- La seconde phase, qui s'appuie sur la précédente, consiste en l'élaboration d'un protocole d'observation ou d'expérimentation permettant l'exploration du champ empirique associé;

- La collecte et le traitement des données issues de la mise en œuvre du protocole constituent la troisième phase;

- Enfin, la quatrième phase consiste à confronter les données recueillies $\mathrm{au}(\mathrm{x})$ modèle(s) et à apporter une réponse argumentée au problème posé. statut du(des) modèle(s) interrogé(s), le type de problème à résoudre, la nature du registre empirique, les compétences visées au travers des tâches demandées aux élèves, les outils mobilisés, la durée relative et le degré d'imbrication éventuel des quatre étapes de l'investigation.

\subsection{L'élaboration d'un référentiel de compétences}

La mise en œuvre d'une démarche d'investigation scientifique dans un contexte d'enseignement précis a conduit les concepteurs à définir un ensemble de compétences disciplinaires ou transversales se traduisant, lors de la mise en œuvre du scénario, par différents types de tâches demandées aux élèves. Ces tâches ont été précisément définies et classées en trois catégories. Elles peuvent porter sur la capacité à s'approprier un modèle, la capacité à articuler le registre du modèle avec le registre empirique et, enfin, les compétences plus particulièrement liées à la maîtrise du registre empirique. Nous distinguons la tâche qui est prescrite à l'élève de l'activité qui est réalisée par l'élève (Pastré, Mayen et Vergnaud, 2006).

\subsection{La prise en compte du contexte de mise en œuvre}

Les deux exemples présentés plus haut diffèrent par la nature des contextes de mise en œuvre. Les contraintes associées sont de plusieurs natures : 


\begin{tabular}{|c|c|}
\hline Statut du(des) modèle(s) & $\begin{array}{ll}\text { - } & \text { admis par la communauté scientifique } \\
\text { - } & \text { ancien(s) et remis en cause par la communauté scientifique } \\
\text { - } & \text { discuté(s) par la communauté scientifique } \\
\text { - } & \text { incomplet (s) pour répondre au problème posé }\end{array}$ \\
\hline $\begin{array}{l}\text { Type de problème à } \\
\text { résoudre }\end{array}$ & $\begin{array}{ll}\text { - } & \text { choisir parmi plusieurs modèles } \\
\text { - } & \text { éprouver un modèle } \\
\text { - } & \text { compléter un modèle partiel } \\
\text { - } & \text { discuter un modèle ancien } \\
\text { - } & \text { confronter plusieurs modèles } \\
\text { - } & \text { instancier un modèle }\end{array}$ \\
\hline Nature du champ empirique & $\begin{array}{ll}\text { - } & \text { le terrain : panorama, affleurement, échantillons... } \\
\text { - } & \text { des images du terrain } \\
\text { - } & \text { l'expérimentation } \\
\text { - } & \text { des résultats d'expérimentation }\end{array}$ \\
\hline Outils et techniques & $\begin{array}{l}\text { - Géonote, SlG } \\
\text { - actiwités de laboratoire : microscope, ExAO } \\
\text { - actiwités de terrain : boussole, GPS, loupe... }\end{array}$ \\
\hline Durée & $\begin{array}{ll}\text { - } & \text { classe de terrain (un à plusieurs jours) } \\
\text { - } & \text { une séance de Travaux Pratiques } \\
- & \text { Travaux Personnels Encadrés : plusieurs séances }\end{array}$ \\
\hline
\end{tabular}

Figure 4. Quelques exemples de critères permettant de différencier les scénarios

- Les contraintes curriculaires liées au respect des instructions officielles du point de vue des démarches à mettre en œuvre, des notions abordées, des savoir-faire développés ou des contraintes horaires. Les expérimentations décrites plus haut respectent ces contraintes;

- Les contraintes disciplinaires liées aux spécificités des objets étudiés. En sciences de la Terre, la nature de ces objets implique que l'enseignant recourt à un travail sur le terrain ou dispose d'images permettant de représenter les caractéristiques du registre empirique qu'il souhaite faire étudier à ses élèves;

- Les contraintes didactiques et pédagogiques liées à la nature du problème que les élèves ont à résoudre et à leur mâ̂trise des concepts nécessaires à sa résolution. Dans les exemples présentés, l'étape d'appropriation du modèle de fonctionnement des plaques lithosphériques est bien différente en classe de quatrième où ce modèle est étudié pour la première fois de celle qui est abordée en classe de terminale où les élèves l'ont mobilisé à plusieurs reprises. Par ailleurs, dans ces deux exemples, la nature du travail d'investigation conduit est différente. En classe de terminale, les élèves éprouvent un modèle de la tectonique des plaques alors qu'en classe de quatrième, ils choisissent parmi plusieurs modèles celui qui correspond à la région étudiée. Ces deux situations d'enseignement mobilisent donc des compétences différentes et conduisent à la mise en œuvre de tâches spécifiques;

- Des contraintes matérielles, liées à la disponibilité des outils nécessaires, dans le contexte de la classe ou dans d'autres lieux tels que la salle de TP, la classe de terrain ou le domicile;

- Des contraintes organisationnelles ou économiques, liées par exemple au coût d'une classe de terrain et à la disponibilité d'une équipe enseignante prête à l'encadrer;

- Des contraintes beaucoup plus difficilement évaluables et associées aux représentations et savoir-faire des enseignants.

La prise en compte de toutes ces contraintes, auxquelles des poids différents sont accordés selon les cas, va déterminer le choix d'une stratégie qui influera fortement sur l'élaboration du scénario. 


\section{Tâches centrées sur l'appropriation du modèle (M)}

M1 Identifier les caractéristiques du modèle

M2 Identifier les propriétés du modèle

Tâches conduisant à la mise en relation du modèle et du registre empirique (EM) Élaboration du protocole d'observation oul d'expérimentation

EM1 Déterminer des implications du modèle en temes d'observables de laboratoire ou de terrain

EM2 Déterminer les procédures du protocole

Confrontation des données empiriques et du modèle scientifique

EM3 Argumenter l'adéquation des données empiriques aux caractéristiques et propriétés du modèle

EM4 Instancier le modèle : le paramétrer, le contextualiser avec des données empiriques

EM5 Définir le domaine de validité du modèle

EM6 Identifier des contraintes (ou nécessités) du modèle

\section{Tâches en relation avec la maitrise du registre empirique (RE)}

RE1 Sélectionner des données empiriques pertinentes au regard de leur lisibilité

RE2 Déteminer les caractéristiques des données empiriques, les situer dans l'espace etiou dans le temps

RE3 Mettre en forme des données empiriques pour faciliter leur lecture

Figure 5. Typologie des tâches mises en œuvre dans une démarche d'investigation

\section{Modéliser le processus d'élaboration} d'un scénario

Dans le cadre des expérimentations décrites plus haut, la formalisation d'un scénario précisant l'organisation des tâches a été nécessaire et a abouti au développement de deux types de supports accessibles en ligne. La fiche-élève fournit un cadre de travail favorisant l'autonomie dans la mesure où, même si des objectifs de réalisation sont annoncés, les élèves ont le choix des stratégies qu'ils adoptent. La fiche-enseignant se présente quant à elle comme un conducteur, au sens donné à ce terme dans l'industrie audiovisuelle, qui permet le pilotage des séances par des enseignants n'ayant pas participé à l'élaboration du scénario. Durant les différentes phases de l'expérimentation, les concepteurs du dispositif ont été conduits à produire des versions successives de ces fiches tenant compte de la variété des contextes. Nous jugeons qu'une meilleure expression des dimensions didactique, pédagogique et situationnelle et de leurs interrelations permettrait d'envisager une adaptation plus facile des scénarios précédemment créés. C'est dans cette perspective d'amélioration des stratégies de réutilisation que nous nous inscrivons : nous jugeons nécessaire d'étudier le processus d'élaboration des scénarios par les enseignants afin d'améliorer les pra- tiques de partage et de diffusion (Pernin, 2007). Certains travaux comparables (Henri et Maina, 2007) s'intéressent à étudier dans ce contexte l'application de méthodes de design pédagogique telles que MISA (Paquette, 2002). Selon notre approche, l'élaboration d'un scénario doit également s'appuyer sur des stratégies de réutilisation de savoir-faire antérieur et d'imitation de bonnes pratiques reconnues ou suggérées, associées à des représentations personnelles du métier de pédagogue et d'expert de la discipline.

Sur la base de l'analyse précédente, nous proposons un processus métier composé de deux phases principales (cf. figure 6).

\subsection{Phase de choix d'une démarche type}

Cette première phase correspond au choix d'une démarche type par le concepteur. Notre première hypothèse (influence de l'approche didactique et pédagogique) repose sur le fait que, lorsqu'il choisit une démarche type, un enseignant s'inscrit implicitement dans le cadre d'une théorie de l'apprentissage et s'appuie sur une représentation de la façon dont l'élève peut construire ses connaissances. Cette approche peut lui être imposée, représenter les valeurs dans lesquelles il se reconnaît implicitement ou bien résulter d'un choix assumé prenant en compte les caractéristiques de la situation d'ap- 


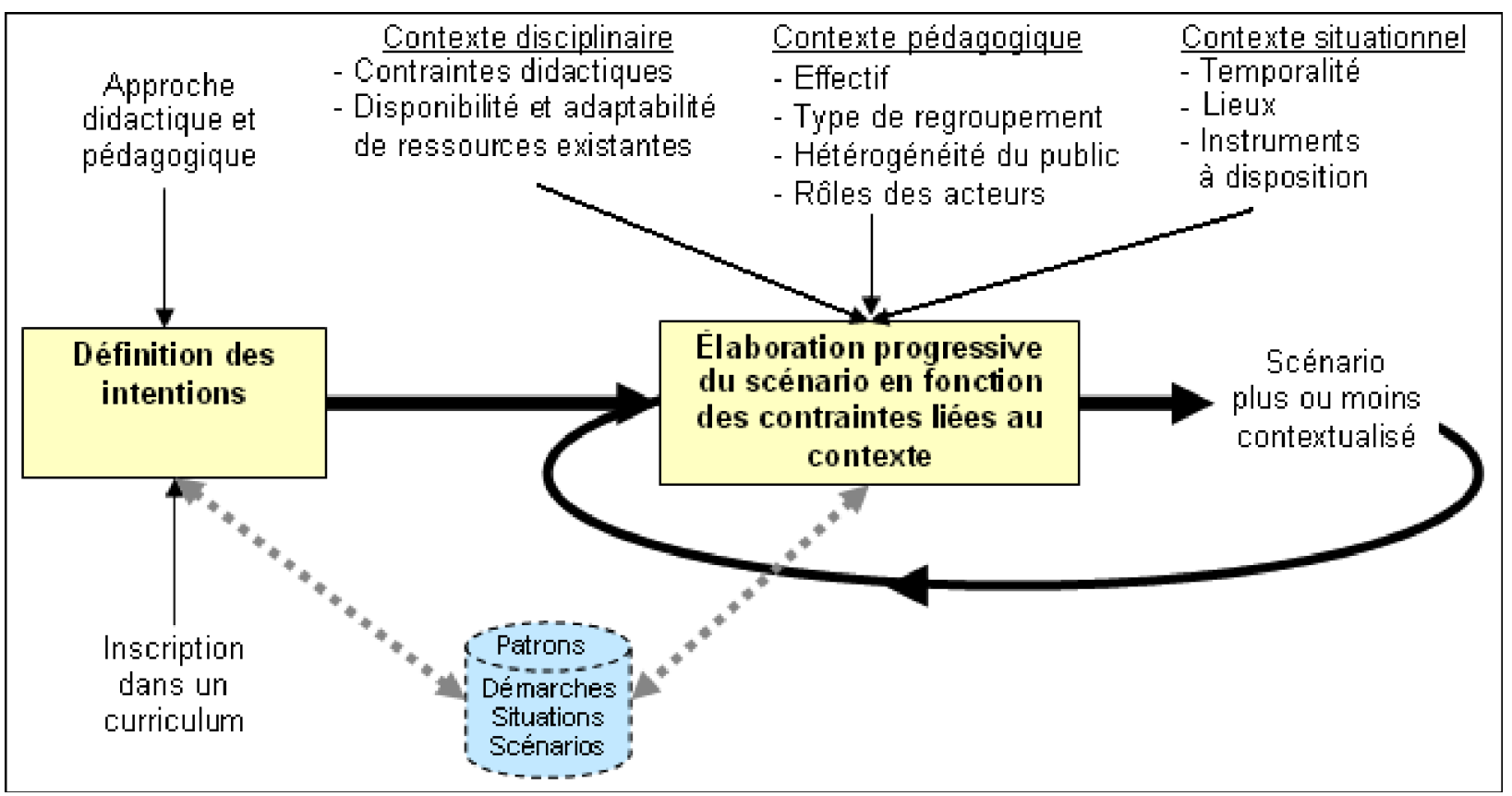

Figure 6. Processus d'élaboration des scénarios

prentissage. Notre seconde hypothèse (inscription dans un curriculum) souligne l'importance des programmes officiels et de l'évaluation, particulièrement mis en avant dans l'enseignement secondaire français :

Les programmes définissent, pour chaque cycle, les connaissances essentielles qui doivent être acquises au cours du cycle ainsi que les méthodes qui doivent être assimilées. Ils constituent le cadre national au sein duquel les enseignants organisent leurs enseignements en prenant en compte les rythmes d'apprentissage de chaque élève (Article L311-3 du Code de l'Éducation cité par le Ministère français de l'Éducation nationale, 2006).

Lorsqu'il choisit une démarche type, un enseignant accorde une place importante aux points du programme et à l'ensemble des compétences disciplinaires ou transversales que les élèves doivent maîtriser. Au sein d'une discipline ou d'un ensemble de disciplines, il existe ainsi des démarches éprouvées pour leur capacité à couvrir les compétences visées. Dans notre cas d'étude, la conduite d'un travail $d^{\prime}$ investigation en sciences s'appuyant sur quatre grandes phases pourra constituer un cadre récurrent donnant lieu à un ensemble de déclinaisons spécifiques.

\subsection{Phase d'élaboration progressive du scénario en fonction des contraintes liées au contexte}

Durant cette phase, le concepteur élabore progressivement son scénario en se fondant sur la démarche type retenue. Cette élaboration repose sur la prise en compte de différents types de contraintes :

- Les contraintes situationnelles relatives aux dimensions spatiale, temporelle ou instrumentale de la situation à mettre en place. Ainsi, suivant les cas, la distribution des activités sera envisagée selon des modalités différentes (dans une salle de travaux pratiques, sur le terrain, à la maison, etc.) et selon des calendriers. La modalité devra également être compatible avec la disponibilité des instruments (logiciels ou non) et/ou des services_nécessaires à la réalisation des tâches;

- Les contraintes didactiques, fréquemment mises en avant par les enseignants du secondaire, influent également sur le choix des modalités retenues. Par exemple, dans le cadre de la conduite d'une démarche d'investigation en sciences de la 
Terre, on pourra opter pour le travail de terrain ou pour une consultation de données virtuelles en fonction de la nature du problème posé;

- Enfin, certaines contraintes économiques ou administratives constituent un élément important du choix d'une stratégie. Ces contraintes peuvent être aussi bien d'ordre financier (ex. : le coût du transport) qu'organisationnel (ex. : la capacité à encadrer une classe de terrain pendant deux jours).

Le scénario élaboré résulterait donc (cf. figure 6) de l'inscription de l'enseignant-concepteur dans un cadre pédagogique et disciplinaire puis d'un processus d'ajustements successifs tenant compte des différentes dimensions de la situation à mettre en place.

\section{Vers l'opérationnalisation du processus : stratégies types et scénarios types}

Le modèle de processus décrit précédemment résulte de l'analyse d'un travail de recherche mené par deux enseignants-concepteurs possédant une maîtrise élevée de la didactique de leur discipline. Cette spécificité a permis de s'appuyer sur une explicitation continue et justifiée des différentes phases du processus (inscription dans un curriculum ou contexte de connaissance), choix d'une démarche didactique, prise en compte progressive des contraintes). Notre hypothèse de recherche est que ce même processus est appliqué, le plus souvent de façon implicite, par des enseignants moins spécialisés, en particulier par le public auquel nous nous intéressons. Ce processus gagnerait donc à être explicité et formalisé au sein même des scénarios afin d'en permettre une meilleure appropriation et d'assurer de meilleures conditions de réutilisation.

Lors des travaux que nous menons dans le cadre du projet CAUSA, nous avons demandé à un groupe-pilote de cinq enseignants de l'enseignement secondaire de rechercher et d'analyser les ressources de type « scénario » que l'on peut trouver sur Internet, pour pouvoir les réutiliser et les intégrer dans leurs disciplines respectives (histoire-géographie, sciences économiques, langues, technologie). Les enseignants avaient pour première consigne d'analyser les stratégies de recherche que les différents recherches permettaient de mettre en œuvre et d'évaluer la pertinence des résultats obtenus vis-à-vis de leurs propres objectifs de conception. Une seconde consigne consistait à demander à chacun de réaliser une synthèse et d'effectuer un ensemble de suggestions permettant d'améliorer le processus de conception. S'il est possible d'observer certaines préoccupations propres à chaque discipline, un ensemble de constats et de propositions communs a été formulé :

- Il existe une très forte hétérogénéité de la terminologie portant sur les scénarios entre les disciplines ou au sein d'une même discipline. Les termes (scénario, phase, activité, séance, séquence, compétence, savoirfaire, thème, etc.) sont rarement clairement définis et rendent souvent complexe l'appropriation de scénarios élaborés par d'autres;

- On ne trouve pas toujours des descriptions de scénario mettant en avant les concepts relevant du champ disciplinaire visé tels que les notions du programme étudiées, les référentiels traités, etc. Cette carence, qui rend difficile la recherche de scénarios éprouvés dans le cadre d'une discipline spécifique, doit être comblée;

- Il est rare, pour un scénario donné, de trouver explicitée la stratégie pédagogique ou didactique associée. Ce manque d'explicitation demande fréquemment une analyse très fine du scénario alors que la stratégie ou la démarche peut constituer un critère de choix très important pour l'enseignant;

- La tâche de conception pourrait être facilitée par la mise à disposition de bibliothèques de stratégies types et de scénarios types, de granularité plus ou moins fine, chacune de ces propositions pouvant être illustrée par des exemples concrets et transposables.

Ces propositions mettent notamment en avant la nécessité d'accompagner l'enseignant-concepteur en lui fournissant des mécanismes d'assistance lors de l'élaboration de scénarios (Villiot-Leclercq, 2007). 


\section{Les stratégies de réutilisation pour la conception de scénarios}

Un des problèmes constatés lors du colloque Scénarios 2007 réside dans la difficulté de mettre en place des stratégies appropriées de partage et de réutilisation pour la conception de scénarios pédagogiques. Des questions spécifiques ont été soulevées : quels éléments réutilisables faut-il mettre à disposition des concepteurs? Comment, dans une perspective de réutilisation ultérieure, abstraire dans un scénario existant ce qui peut être ou non réutilisable? Comment le concepteur peut-il retrouver une solution ou des éléments de solution lui permettant de proposer un scénario adapté à ses objectifs et à son contexte particulier?

Afin d'éclairer cette problématique, nous fournissons dans cette contribution un ensemble de critères permettant de distinguer les différentes approches de réutilisation de scénarios. Nous proposons ainsi de définir plus précisément 1) les stratégies de partage des objets appelés à être réutilisés; 2) les stratégies de réutilisation de ces objets par les concepteurs; et 3) les stratégies de recherche d'objets dans une banque de données.

\subsection{Stratégies de partage d'objets réutilisables}

Parmi les travaux actuels visant la mise en place des mécanismes de mutualisation de scénarios pédagogiques, deux approches peuvent être distinguées. La première, illustrée par le projet IDLD (Lundgren-Cayrol, Marino, Paquette, Léonard et De La Teja, 2006), propose des banques de scénarios notamment modélisés dans un langage de notation tel qu'IMSLD et indexés à l'aide d'un jeu spécifique de métadonnées LOM pour les objets d'apprentissage. L'objectif principal est de permettre la réutilisation d'un scénario modélisé selon un langage de notation interopérable pour l'adapter et le mettre en œuvre dans un contexte technique différent du premier. Si ces travaux sont indispensables pour assurer la réutilisation dans le cadre de l'ingénierie de formation à distance, il semble nécessaire de les enrichir pour les rendre manipulables par les enseignants dans un cadre académique. Une seconde approche consiste donc à mettre en place des banques de pratiques pour les enseignants, telles que les bases proposées par le ministère de l'Éducation (EDU'bases) (PrimTICE). Les scénarios y sont indexés avec des champs différents selon les disciplines et leur description peut prendre des formes très variables allant de simples narrations de pratique jusqu'à des formalisations proches des langages de notation (Macedo et Perron, 2007).

Les objets de type scénario mis à disposition sont essentiellement de deux types. On peut ainsi trouver des scénarios instanciés décrivant les différentes facettes de la situation d'apprentissage à mettre en place. La mutualisation de ces scénarios fortement contextualisés résulte en général d'un processus de validation émanant d'une autorité qui en vérifie le caractère « exemplaire ». C'est par exemple le processus choisi par les banques de pratiques EDU'bases ou PrimTICE qui sont validées au niveau académique.

On peut également trouver des gabarits de scénarios proposant des ossatures à partir desquelles peuvent être dérivées des solutions différentes selon les contextes; c'est également un des objectifs du répertoire IDLD ou d'outils spécifiques de conception de scénarios tels que l'outil d'édition COLLAGE, qui permet la création de scénarios collaboratifs à partir d'un catalogue de patrons prédéfinis (Hernández-Leo et al., 2006).

On peut également imaginer de disposer de répertoires de composants permettant de structurer l'organisation et le déroulement d'un scénario à des niveaux plus ou moins élevés de granularité. De tels composants pourraient être agencés ou agrégés pour constituer une solution adaptée au besoin d'un concepteur en fonction de son contexte. C'est par exemple la démarche proposée par la méthode des Pléiades (Villiot-Leclercq, 2007), qui propose une métaphore astronomique (concepts de constellation, de pléiades et d'étoiles) pour soutenir la démarche de conception.

\subsection{Stratégie de réutilisation des objets}

Pour un concepteur donné, plusieurs stratégies peuvent être mises en œuvre pour élaborer un scénario adapté à ses 
objectifs et à son contexte à partir d'objets retrouvés dans des répertoires spécialisés. Trois grandes classes de stratégies, liées étroitement aux types d'objets décrits ci-dessus, peuvent ainsi être définies.

Les stratégies d'imitation sont les plus couramment mises en œuvre à partir de «solutions éprouvées ». Elles consistent à s'inspirer de scénarios « exemplaires » instanciés dans un contexte précis. La difficulté pour l'utilisateur vient alors de la nécessité de distinguer au sein du scénario, d'une part ce qui relève du contexte initial de mise en œuvre, et d'autre part ce qui relève d'invariants pouvant être réappliqués dans de nouveaux contextes. Dans ce cas, un important travail d'appropriation, de décontextualisation puis d'adaptation doit être effectué.

Les stratégies d'instanciation se fondent sur la réutilisation de gabarits qui ont été élaborés le plus souvent à partir de solutions dont on a pu établir le caractère transférable. Le travail du concepteur revient ici à choisir le gabarit le mieux adapté à son contexte pour ensuite en décrire les particularités propres. La complexité réside alors davantage dans la recherche du gabarit approprié que dans ses possibilités d'adaptation, qui ont été a priori prévues lors de l'élaboration du gabarit.

Enfin, les stratégies de composition consistent, pour le concepteur, à agréger des composants retrouvés dans des répertoires pour construire une solution adaptée à ses besoins propres. Dans ce cas, il est nécessaire de s'appuyer sur un modèle conceptuel structurant précisant les relations entre les différents composants : le modèle doit être suffisamment «communicable » pour être partagé par un ensemble identifié de concepteurs. C'est un des objectifs poursuivis par la méthode des Pléiades, qui propose une métaphore pour représenter différentes granularités de scénarios : une activité élémentaire est une étoile, un regroupement de plusieurs activités est une pléiade, un regroupement de plusieurs regroupements ou pléiades est une constellation (Villiot-Leclercq, 2007). C'est également la voie que nous explorons actuellement au travers du développement $d$ 'un environnement de conception reposant sur un modèle conceptuel issu des réflexions présentées dans cet article. Ce modèle ISiS décompose la conception d'un scénario comme une imbrication de trois niveaux successifs : expression des intentions (I) du concepteur, des démarches ou des stratégies (S) qu'il adopte, puis des situations d'interaction (iS) qu'il sélectionne pour répondre à ses exigences.

\subsection{Stratégie de recherche des objets}

Un dernier critère concerne le mode de recherche d'objets réutilisables qui, comme nous l'avons vu, peuvent être soit des scénarios complets, soit des ossatures de scénarios, soit des composants de scénarios. Deux grands types de modalités de recherche sont envisageables :

Les stratégies classiques de recherche reposent sur des techniques d'indexation d'éléments à l'aide de mots-clés ou de métadonnées. Elles permettent à l'utilisateur de retrouver le type d'objet qu'il recherche après avoir formulé une requête précisant un nombre variable de critères. Ce type de démarche, adoptée par les répertoires tels qu'EDU'base, PrimTICE ou IDLD, se heurte à un écueil fréquemment identifié en systèmes d'information : difficulté à trouver un équilibre entre une indexation trop précise et une recherche efficace.

Une autre approche consiste à s'appuyer sur des patrons de conception (design patterns) (Alexander, Ishikawa et Silverstein, 1977) pour proposer au concepteur des solutions éprouvées pour un problème récurrent dans un contexte donné. C'est la solution que nous préconisons dans l'environnement de conception que nous réalisons actuellement pour opérationnaliser le processus de conception présenté dans cet article. En l'occurrence, il s'agira pour le concepteur de retrouver des composants en fonction de l'étape du processus dans laquelle il se trouve et qu'il pourra intégrer progressivement. Ainsi, le concepteur pourra notamment, en tenant explicitement compte d'éléments du contexte, 1) retrouver des stratégies ou des démarches didactiques adaptées aux intentions qu'il veut mettre en œuvre; et 2) retrouver des situations types d'interaction adaptées aux stratégies retenues. 


\section{Conclusion et perspectives}

Nous avons proposé dans cet article un processus métier d'élaboration des scénarios pédagogiques reposant sur deux phases principales; la phase de choix d'une démarche type dirigée par les intentions est suivie d'une phase d'affinage progressif du scénario prenant en compte les différentes dimensions du contexte. Cette vision est à rapprocher de récents travaux de recherche menés dans le champ du génie logiciel concernant l'ingénierie des processus dirigée par les intentions (Rolland, 2000). Il nous semble nécessaire d'exploiter les résultats déjà obtenus dans ce champ et de contribuer à un enrichissement des réflexions en appliquant ces recherches au domaine des environnements informatiques pour l'apprentissage humain.

$C^{\prime}$ est dans cette perspective que nous menons actuellement, dans le cadre du projet CAUSA, un travail de développement $\mathrm{d}$ 'un environnement informatique permettant la mise en œuvre du processus métier présenté dans cet article. Grâce à cet environnement, l'enseignantconcepteur pourra :

- préciser l'approche pédagogique dans laquelle il s'inscrit;

- déterminer les référentiels disciplinaires ou transversaux auxquels il se rattache;

- définir les contraintes didactiques, situationnelles, organisationnelles ou techniques associées aux contextes de mise en œuvre qui l'intéressent;

- explorer un ensemble de démarches types compatibles avec l'approche pédagogique retenue et/ou les référentiels sélectionnés;

- explorer de façon souple des scénarios de mise en œuvre basés sur la démarche type retenue, répondant à l'ensemble ou une partie des contraintes définies;

- disposer d'une description suffisamment formalisée des scénarios pour les rendre adaptables tout en assurant leur capacité à être opérationnalisés à moindre coût vers des plateformes de formation.

Ce développement s'effectue en parallèle à la réalisation de gabarits de démarches types issus à la fois de la littérature et des pratiques des enseignants. Nous avons également pour objectif de valider les hypothèses concernant le processus métier décrit en proposant aux enseignants un panel de stratégies d'indexation et de recherche le plus large possible.

\section{Références}

Alexander, C., Ishikawa, S. et Silverstein, M. (1977). A pattern language: Towns, buildings, construction. New York: Oxford University Press.

Artigue, M. (1988). Ingénierie didactique. Recherches en didactique des mathématiques, 9(3), 281-308.

Bachelard, S. (1979). Quelques aspects historiques des notions de modèle et de justification des modèles. Dans P. Delattre et M. Thellier (dir.), Élaboration et justification des modèles (tome I, p. 3-19). Paris : Maloine.

Bunge, M. (1975). Philosophie de la physique. Paris : Seuil.

Halloun, I. A. (2004). Modeling theory in science education. Dordrecht, Pays-Bas : Kluwer Academic.

Henri, F. et Maina, M. (2007). Pratique de design pédagogique et instrumentation du concepteur. Dans M. Baron, D. Guin et L. Trouche (dir.), Environnements informatisés et ressources numériques pour l'apprentissage : conception et usages, regards croisés (p. 229-254). Paris : Hermès.

Hernández-Leo, D., Villasclaras-Fernández, E. D., Asensio-Pérez, J. I., Dimitriadis, Y., Jorrín-Abellán, I. M., Ruiz-Requies, I. et al. (2006). COLLAGE: A collaborative learning design editor based on patterns. Educational Technology \& Society, 9(1), 58-71.

Lefèvre, O. et Sanchez, E. (2006, avril). Géonote : un environnement informatique d'aide au travail sur le terrain pour l'enseignement des sciences de la terre. Communication présentée à la 8e Biennale de l'éducation et de la formation, Lyon, France.

Lundgren-Cayrol, K., Marino, O., Paquette, G., Léonard, M. et De La Teja, I. (2006). Implementation and deployment process of IMS learning design: Findings from the Canadian IDLD research project. Dans Proceedings of the Sixth IEEE International Conference on Advanced Learning Technologies (p. 581-585). Washington, DC : IEEE Computer Society.

Macedo, M. et Perron, J.-M. (2007). Caractérisation des scénarios pédagogiques utilisant les TICE. Dans Actes de la conférence Environnement informatique pour l'apprentissage humain (EIAH 07) (p. 101-112). Paris/Lyon : Association des technologies de l'information pour l'éducation et la formation [ATIEF] et Institut national de recherche pédagogique [INRP]. 
Ministère français de l'Éducation nationale. (2006, 25 septembre). ÉduSCOL - Les programmes scolaires. Récupéré le 1er décembre 2007 du site ÉduScol, section Projet de programmes de l'école primaire Programmes par discipline: http:/ / eduscol.education.fr/D0048/ LLPPRC01.htm

Paquette, G. (2002). L'ingénierie pédagogique: pour construire l'apprentissage en réseau. Sainte-Foy, Canada : Presses de l'Université du Québec.

Pastré, P., Mayen, P. et Vergnaud, G. (2006). La didactique professionnelle. Revue française de pédagogie, 154, 145-198.

Pernin, J.-P. (2007). Mieux articuler activités pour l'apprentissage, artefacts logiciels et connaissances : vers un modèle d'ingénierie centré sur le concept de scénario. Dans M. Baron, D. Guin et L. Trouche (dir.), Environnements informatisés et ressources numériques pour l'apprentissage: conception et usages, regards croisés (p. 161-190). Paris : Hermès.

Pernin, J.-P. et Emin, V. (2006). Évaluation des pratiques de scénarisation de situations d'apprentissage : une première étude. Informations, savoirs, décisions et médiations, 25, article 359. Récupéré du site de la revue : http://isdm.univ-tln.fr/PDF/isdm25/PerninEmin TICE2006.pdf

Rolland, C. (2000). Intention driven component reuse. Dans S. Brinkkemper, E. Lindecrona et A. Solvberg (dir.), Proceedings of the Workshop on Information Systems Engineering (p. 197-208). Stockholm : Springer.

Sanchez, E. et Prieur, M. (2006). Démarche d'investigation dans l'enseignement des sciences de la Terre : activités-élèves et scénarios. Dans J.-P. Pernin et H. Godinet (dir.), Actes du colloque Scénariser l'enseignement et l'apprentissage : une nouvelle compétence pour le praticien? Colloque réalisé dans le cadre de la 8e Biennale de l'éducation et de la formation (p. 71-76). Lyon, France : Institut national de recherche pédagogique [INRP]. Récupéré du site de l'institut, section Publications -Édition électronique : http:/ / www.inrp. $\mathrm{fr} /$ INRP/publications/ editions-electroniques

Tricot, A., Plégat-Soutjis, F., Camps, J.-F., Amiel, A., Lutz, G. et Morcillo, A. (2003). Utilité, utilisabilité, acceptabilité : interpréter les relations entre trois dimensions de l'évaluation des EIAH. Dans C. Desmoulins, P. Marquet et D. Bouhineau (dir.), Actes de la conférence Environnements Informatiques pour l'Apprentissage Humain (EIAH 2003) (p. 391-402). Paris/Lyon : Association des technologies de l'information pour l'éducation et la formation [ATIEF] et Institut national de recherche pédagogique [INRP].
Villiot-Leclercq, E. (2007). Modèle de soutien pour l'élaboration et la réutilisation de scénarios pédagogiques. Thèse de doctorat non publiée, Université Joseph Fourier/Université de Montréal, France/Canada. Walliser, B. (1977). Systèmes et modèles. Paris : Seuil. 